\title{
SYNTHESIS AND REACTIONS OF [1]BENZOFURO[3,2-c]PYRIDINE
}

\author{
NADEŽDA JURISTOVÁ ${ }^{1}$, ELEONÓRA ŠTEFANOVOVÁ ${ }^{1}$, \\ TATIANA ĎURČEKOVÁ ${ }^{1}$, NAĎA PRÓNAYOVÁ ${ }^{2}$, ANTON \\ GATIAL $^{3}$, ALŽBETA KRUTOŠÍKOVÁ $^{1}$ \\ ${ }^{1}$ Department of Chemistry, Faculty of Natural Sciences, University of SS. Cyril and \\ Methodius, SK-917 01 Trnava, Slovak Republic \\ e-mail: alzbeta.krutosikova@ucm.sk \\ ${ }^{2}$ Institute of Analytical Chemistry, Department of NMR and MS Spectroscopy, Slovak \\ University of Technology, Radlinského 9, SK-812 37 Bratislava, Slovak Republic \\ ${ }^{3}$ Institute of Physical Chemistry and Chemical Physics, Slovak University of \\ Technology, Radlinského 9, SK-812 37 Bratislava, Slovak Republic
}

\begin{abstract}
E)-3-(1-Benzofuran-2-yl)propenoic acid (I) was prepared from 1-benzofuran-2-carbaldehyde under the Doebner's conditions. The obtained acid was converted to the corresponding azide $I I$, which was cyclized by heating in diphenyl ether to [1]benzofuro[3,2-c]pyridin-1(2H)-one (III). This compound was aromatized with phosphorus oxychloride to chloroderivative $I V$ which was reduced with zinc and acetic acid to the title compound $V$. [1]Benzofuro[3,2-c]pyridin-2-oxide (VI) was synthesized by reaction of $V$ with 3chloroperoxybenzoic acid in dichloromethane. Treatment VI with benzoyl chloride and potassium cyanide (Reissert-Henze reaction) was shown to produce the corresponding [1]benzofuro[3,2-c]pyridin-1carbonitrile (VII). The title compound was used for preparation of complex compounds VIII, IX
\end{abstract}

Key words: [1]Benzofuro[3,2-c]pyridine, $N$-oxide, 1-carbonitrile, ${ }^{1} \mathrm{H},{ }^{13} \mathrm{C}$ NMR and IR spectra, metalorganic compounds

\section{Introduction}

Furopyridines are of chemical interest due to their similarity to quinoline, isoquinoline, and benzofuran, which are important ring systems present in many biologically active compounds. The antihypertensitive drug cicletanine is a furo[3,2c]pyridine derivative. The furopyridine skeleton from which this compound is derived is not associated with any known pharmacological classes (SHERMAN, 1996).

In the past one of us has been interested in studying the synthesis and reactivity of various furo[3,2-c]pyridines (BOBOŠÍK et al., 1995; KRUTOŠÍKOVÁ et al., 1992, 1994, 1995; KRUTOŠÍKOVÁ and SLEZIAK, 1996). The authors (BENCKOVÁ and KRUTOŠÍKOVÁ, 1995; 1999) were concentrated on transformations on the pyridine ring of some of this type of compounds. Later on some furo[3,2-c]pyridines have been used for preparation $\mathrm{Cu}(\mathrm{II})$ and $\mathrm{Ni}(\mathrm{II})$ complexes (KRUTOŠÍKOVÁ et al., 2001). Furo[3,2-c]pyridine and its 2-methyl-, 2,3-dimethyl and benzo[4,5] derivative were used for the first time as ligands to synthesize potentially new Werner clathrates and their structural characterization, spectral and magnetic properties of isothiocyanate nickel(II) complexes (MIKLOVIČ et al., 2004, MOJUMDAR et al., 2005). Magneto structural correlations in heteroleptic furo[3,2-c]pyridineNi(II) complexes were 
published by the research group (BARAN et al., 2005). Recently two papers dealing about the syntheses and crystal structures of tetra- $\mu$-acetato-bis(1-benzofuro[3,2$c$ ]pyridinecopper(II) and bis(1-benzofuro[3,2-c]pyridine- $\kappa N)$ dichlorocobalt(II) were published (VRABEL et al., 2007).

In this paper, which continues our previous papers (BENCKOVÁ and KRUTOŠÍKOVÁ, 1999; KRUTOŠÍKOVÁ et al., 1992, 1994, 1995) we report the synthesis of [1]benzofuro[3,2-c]pyridine and its 2-oxide (Scheme 1).

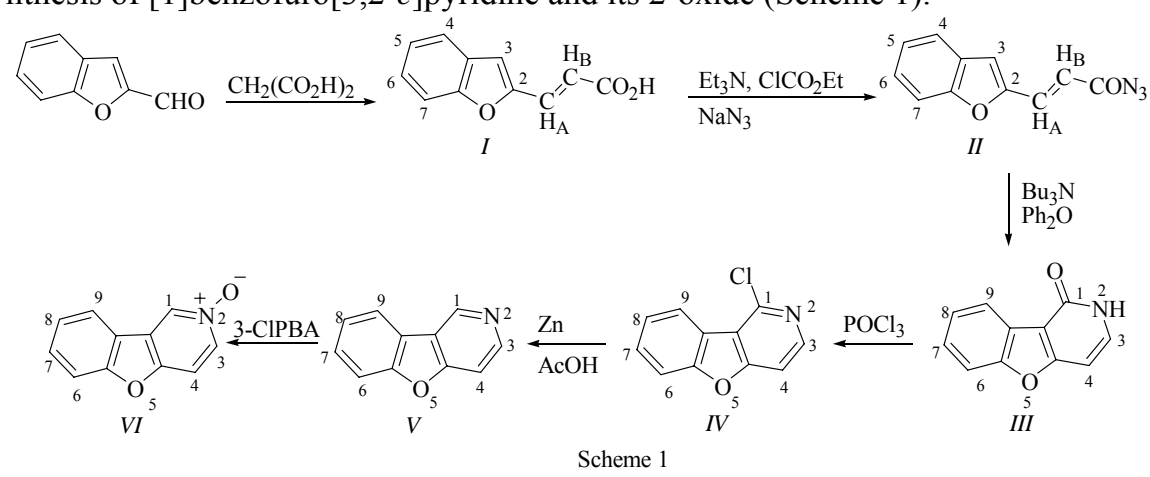

\section{Experimental}

Melting points were determined using Kofler hot plate. All solvents were distilled and dried before use. All reagents were commercially available and were used without purification. Elemental analyses were determined using an EAGER 300 at Institute of Inorganic Chemistry, Technology and Materials STU in Bratislava. IR spectra were taken on a FTIR Nicolet NEXUS 470 spectrophotometer using $\mathrm{KBr}$ technique $(0.5 \mathrm{mg}$ in $300 \mathrm{mg} \mathrm{KBr}$ ) in region $4000-400 \mathrm{~cm}^{-1}$ at Institute of Physical Chemistry and Chemical Physics, STU in Bratislava. For interpretation of IR spectra following abbreviations are used: $\mathrm{m}=$ medium band (a value of transmittance: $36-50 \%$ ) $\mathrm{w}=$ weak (a value of transmittance: over 50\%), without marking are strong bands (a value of transmittance: $0-35 \%) .{ }^{1} \mathrm{H}$ NMR spectra were measured in DMSO- $d_{6}$ using Varian INOVA 600 (for ${ }^{1} \mathrm{H} 599,782 \mathrm{MHz}$ and for ${ }^{13} \mathrm{C} 150.830 \mathrm{MHz}$ ) spectrometer at Institute of Analytical Chemistry, Department of NMR and MS Spectroscopy, STU in Bratislava. Chemical shifts ( $\delta$-scale) are quoted in parts per million and following abbreviations are used: $\mathrm{s}=$ singlet; $\mathrm{d}=$ doublet; $\mathrm{t}=$ triplet; $\mathrm{q}=$ quartet; $\mathrm{m}=$ multiplet, coupling constants $(J)$ are given in $\mathrm{Hz}$.

\section{1 (2E)-3-(1-Benzofuran-2-yl)propenoic Acid (I)}

A mixture of 1-benzofuran-2-carbaldehyde (5.1 g; $35 \mathrm{mmol})$, malonic acid (3.7 g; $35 \mathrm{mmol})$, pyridine $\left(39.3 \mathrm{~cm}^{3}\right)$ and piperidine (17 drops) was heated on steam bath for $8 \mathrm{~h}$. The reaction mixture was poured on ice, acidified with $0.5 \mathrm{M}$ hydrochloric acid. The separate precipitate was filtered off and crystallized. Yield: $11.74 \mathrm{~g}(88.9 \%)$; white crystals, m.p. $222-223{ }^{\circ} \mathrm{C}$ (ethanol). For $\mathrm{C}_{11} \mathrm{H}_{7} \mathrm{~N}_{3} \mathrm{O}_{2}\left(M_{r}=188.18\right) w_{\mathrm{i}}$ (calc.): $70.21 \%$ C, $4.29 \% \mathrm{H}$; $w_{\mathrm{i}}$ (found): $70.41 \% \mathrm{C}, 4.26 \% \mathrm{H}$. 
${ }^{1} \mathrm{H}$ NMR spectrum: 12.6 ( bs $\left.\mathrm{CO}_{2} \mathrm{H}\right) ; 7.69\left(\mathrm{~d},{ }^{3} \mathrm{~J}(4,5)=7.8 \mathrm{~Hz},{ }^{4} \mathrm{~J}(4,6)=1.2 \mathrm{~Hz}, \mathrm{H} 4\right)$; $7.59\left(\mathrm{dd},{ }^{3} J(7,6)=8.1 \mathrm{~Hz},{ }^{4} J(7,5)=1.2 \mathrm{~Hz}, \mathrm{H} 7\right) ; 7.57\left(\mathrm{~d},{ }^{3} J(\mathrm{~B}, \mathrm{~A})=15.6 \mathrm{~Hz}, \mathrm{H}_{\mathrm{B}}\right)$; $7.39\left(\mathrm{t},{ }^{3} \mathrm{~J}(6,7)=7.8 \mathrm{~Hz},{ }^{4} \mathrm{~J}(6,4)=1.2 \mathrm{~Hz}, \mathrm{H} 6\right) ; 7.34(\mathrm{~s}, \mathrm{H} 3) ; 7.28\left(\mathrm{t},{ }^{3} \mathrm{~J}(5,6)=7.8 \mathrm{~Hz}\right.$, $\left.{ }^{4} J(5,7)=1.2 \mathrm{~Hz}, \mathrm{H} 5\right) ; 6.41\left(\mathrm{~d},{ }^{3} J(\mathrm{~A}, \mathrm{~B})=15.6 \mathrm{~Hz}, \mathrm{H}_{\mathrm{A}}\right)$.

${ }^{13} \mathrm{C}$ NMR spectrum: $167.1(\mathrm{C}=\mathrm{O}) ; 154.9(\mathrm{C} 7 \mathrm{a}) ; 152.1(\mathrm{C} 2) ; 131.2\left(\mathrm{C}_{\mathrm{B}}\right) ; 128.1(\mathrm{C} 3 \mathrm{a})$; 126.7 (C6); 123.6 (C5); $122.2(\mathrm{C} 4) ; 119.4\left(\mathrm{C}_{\mathrm{A}}\right) ; 111.7(\mathrm{C} 3) ; 111.4(\mathrm{C} 7)$.

IR spectrum: $1671 ; 1630 ; 1451 ; 1419 ; 1304 ; 1268 ; 1200 ; 1127 ; 1007 \mathrm{w} ; 978 \mathrm{w} ; 951$; $882 \mathrm{w} ; 834 \mathrm{~m} ; 753 ; 738 ; 664 \mathrm{w} ; 568 \mathrm{~m} ; 456 \mathrm{w}$.

\section{2 (2E)-3-(1-Benzofuran-2-yl)propenoyl Azide (II)}

Propenoic acid $I(3.76 \mathrm{~g} ; 20 \mathrm{mmol})$ suspended in dry acetone $\left(40 \mathrm{~cm}^{3}\right)$ was cooled to $-10{ }^{\circ} \mathrm{C}$ and triethylamine $\left(3.2 \mathrm{~cm}^{3}\right)$ in dry acetone $\left(5 \mathrm{~cm}^{3}\right)$ was added. Then solution of ethyl chloroformate $\left(2.4 \mathrm{~cm}^{3}\right)$ in dry acetone $\left(5 \mathrm{~cm}^{3}\right)$ was added dropwise to the stirred reaction mixture at temperature lower than $0{ }^{\circ} \mathrm{C}$. The reaction mixture was stirred for another $30 \mathrm{~min}$ at the same temperature. Solution of sodium azide $(2.0 \mathrm{~g}$; $30.8 \mathrm{mmol})$ in water $\left(10 \mathrm{~cm}^{3}\right)$ was added at temperature below $0{ }^{\circ} \mathrm{C}$. The mixture was stirred for an additional hour and allowed to warm to room temperature, poured onto crushed ice and precipitate was filtered off, washed with water and crystallized. Yield: $4.07 \mathrm{~g} ;(95.6 \%)$, m.p. $114-116{ }^{\circ} \mathrm{C}$ white crystals. For $\mathrm{C}_{11} \mathrm{H}_{7} \mathrm{~N}_{3} \mathrm{O}_{2}\left(M_{r}=213.19\right) w_{\mathrm{i}}$ (calc.): $61.97 \% \mathrm{C}, 3.31 \% \mathrm{H}, 19.71 \% \mathrm{~N}$; $w_{\mathrm{i}}$ (found): $61.82 \% \mathrm{C}, 3.27 \% \mathrm{H}, 19.78 \% \mathrm{~N}$.

${ }^{1} \mathrm{H}$ NMR spectrum: $7.74\left(\mathrm{~d},{ }^{3} \mathrm{~J}(\mathrm{~B}, \mathrm{~A})=15.5 \mathrm{~Hz}, \mathrm{H}_{\mathrm{B}}\right) ; 7.73\left(\mathrm{dd},{ }^{3} \mathrm{~J}(4,5)=8.2 \mathrm{~Hz},{ }^{4} \mathrm{~J}(4,6)\right.$ $=1.2 \mathrm{~Hz}, \mathrm{H} 4) ; 7.61\left(\mathrm{dd},{ }^{3} J(7,6)=8.2 \mathrm{~Hz},{ }^{4} J(7,5)=0.88 \mathrm{~Hz}, \mathrm{H} 7\right) ; 7.50(\mathrm{~s}, \mathrm{H} 3) ; 7.44(\mathrm{t}$, $\left.{ }^{3} \mathrm{~J}(6,5)=7.2 \mathrm{~Hz},{ }^{4} \mathrm{~J}(6,4)=1.2 \mathrm{~Hz}, \mathrm{H} 6\right) ; 7.30\left(\mathrm{t},{ }^{3} \mathrm{~J}(5,6)=7.38 \mathrm{~Hz},{ }^{4} \mathrm{~J}(5,7)=0.88 \mathrm{~Hz}\right.$, $\mathrm{H} 5) ; 6.46\left(\mathrm{~d},{ }^{3} \mathrm{~J}(\mathrm{~A}, \mathrm{~B})=15.5 \mathrm{~Hz}, \mathrm{H}_{\mathrm{A}}\right)$.

${ }^{13} \mathrm{C}$ NMR spectrum: $170.9(\mathrm{C}=\mathrm{O}) ; 155.2(\mathrm{C} 7 \mathrm{a}) ; 151.5(\mathrm{C} 2) ; 133.1\left(\mathrm{C}_{\mathrm{B}}\right) ; 127.9(\mathrm{C} 3 \mathrm{a})$; 127.4 (C6); 123.7 (C5); $122.5(\mathrm{C} 4) ; 118.6\left(\mathrm{C}_{\mathrm{A}}\right) ; 114.2$ (C3); $111.4(\mathrm{C} 7)$.

IR spectrum: $2150 ; 1677 ; 1619 ; 1544 ; 1471 ; 1448 ; 1348 ; 1330 ; 1272 ; 1169 ; 1127$; 1096; 999; 951; 824; 788; 687; 630; 610m; 544m; 520m; 441m; 402m;

\section{3 [1]Benzofuro[3,2-c]pyridin-1(2H)-one (III)}

Azide $I I$ ( $8.67 \mathrm{~g}$; $41 \mathrm{mmol})$ dissolved in toluene $\left(67 \mathrm{~cm}^{3}\right)$ was added dropwise into diphenyl ether $\left(35 \mathrm{~cm}^{3}\right)$, and tributylamine $\left(10.7 \mathrm{~cm}^{3}\right)$ were at $180-200{ }^{\circ} \mathrm{C}$ for $45 \mathrm{~min}$, while toluene was distilled out. Then the reaction mixture was heated up $215^{\circ} \mathrm{C}$ for 15 min. After cooling, diethyl ether was added the precipitate was filtered off and washed with diethyl ether. Yield: $6.22 \mathrm{~g}(82.6 \%)$, m.p. $207-209{ }^{\circ} \mathrm{C}$ (ethanol) white crystals.

For $\mathrm{C}_{11} \mathrm{H}_{7} \mathrm{NO}_{2}\left(M_{r}=185.18\right) w_{\mathrm{i}}$ (calc.): $71.35 \% \mathrm{C}, 3.81 \% \mathrm{H}, 7.56 \% \mathrm{~N}$; $w_{\mathrm{i}}$ (found): $71.22 \% \mathrm{C}, 3.78 \% \mathrm{H}, 7.66 \% \mathrm{~N}$.

${ }^{1} \mathrm{H}$ NMR spectrum: 11.83 (bs, NH); $8.01\left(\mathrm{dd},{ }^{3} J(9,8)=7.33 \mathrm{~Hz},{ }^{4} J(9,7)=1.76 \mathrm{~Hz}\right.$, H9); $7.678\left(\mathrm{dd},{ }^{3} J(6,7)=7.04 \mathrm{~Hz},{ }^{4} J(6,8)=1.76 \mathrm{~Hz}, \mathrm{H} 6\right) ; 7.41\left(\mathrm{t},{ }^{3} J(7,8)=7.33 \mathrm{~Hz}\right.$, $\left.{ }^{4} J(7,9)=1.47 \mathrm{~Hz}, \mathrm{H} 7\right) ; 7.407\left(\mathrm{t},{ }^{3} J(8,7)=7.33 \mathrm{~Hz},{ }^{4} J(8,6)=1.47 \mathrm{~Hz}, \mathrm{H} 8\right) ; 7.56(\mathrm{~d}$, $\left.{ }^{3} J(3,4)=7.34 \mathrm{~Hz}, \mathrm{H} 3\right) ; 6.76\left(\mathrm{~d},{ }^{3} J(4,3)=7.34 \mathrm{~Hz}, \mathrm{H} 4\right)$.

${ }^{13} \mathrm{C}$ NMR spectrum: 162.5 (C4a); 159.5 (C1); 154.1 (C5a); 135.2 (C3); 125.7 (C7); 124.2 (C8); 123.6 (C9a); 120.8 (C9); 111.3 (C6); 110.0 (C9b); 94.4 (C4). 
IR spectrum: $1647 ; 1565 ; 1486 ; 1453 ; 1428 ; 1389 ; 1388 \mathrm{~m} ; 1258 \mathrm{~m} ; 1233 ; 1183 ; 1068$; 1000m; 947m; 906m; 846m; 788; 760; 677m; 653m; 587; 543; 43.

\subsection{1-Chloro[1]benzofuro[3,2-c]pyridine (IV)}

A pyridone $I I I$ ( $4.5 \mathrm{~g} ; 24 \mathrm{mmol})$ was refluxed in phosphorus oxychloride $\left(8.6 \mathrm{~cm}^{3}\right)$ for $4 \mathrm{~h}$. $\mathrm{POCl}_{3}$ was removed at reduced pressure and the ice was added to the residue. The mixture was then made basic with diluted aqueous ammonia. The precipitate was filtered out, washed with water, dried and crystallized. Yield: $4.71 \mathrm{~g}(91.2 \%)$, m.p. 65$67{ }^{\circ} \mathrm{C}$ (hexane) white crystals.

For $\mathrm{C}_{11} \mathrm{H}_{6} \mathrm{CINO}\left(M_{r}=203.62\right) w_{\mathrm{i}}$ (calc.): $64.88 \% \mathrm{C}, 2.97 \% \mathrm{H}, 6.88 \% \mathrm{~N} ; w_{\mathrm{i}}$ (found): $64.73 \% \mathrm{C}, 2.95 \% \mathrm{H}, 6.79 \% \mathrm{~N}$.

${ }^{1} \mathrm{H}$ NMR spectrum: $8.44\left(\mathrm{~d},{ }^{3} J(3,4)=5.57 \mathrm{~Hz}, \mathrm{H} 3\right) ; 8.16\left(\mathrm{dd},{ }^{3} J(9,8)=7.8 \mathrm{~Hz},{ }^{4} J(9,7)\right.$ $=1.8 \mathrm{~Hz}, \mathrm{H} 9) ; 7.798\left(\mathrm{~d},{ }^{3} \mathrm{~J}(4,3)=5.57 \mathrm{~Hz}, \mathrm{H} 4\right) ; 7.782\left(\mathrm{dd},{ }^{3} \mathrm{~J}(6,7)=8.3 \mathrm{~Hz},{ }^{4} \mathrm{~J}(6,8)=\right.$ $0.88 \mathrm{~Hz}, \mathrm{H6}) ; 7.64\left(\mathrm{t},{ }^{3} J(7,8)=7.6 \mathrm{~Hz},{ }^{4} J(7,9)=1.8 \mathrm{~Hz}, \mathrm{H} 7\right) ; 7.50\left(\mathrm{t},{ }^{3} J(8,7)=7.6 \mathrm{~Hz}\right.$, $\left.{ }^{4} J(8,6)=0.88 \mathrm{~Hz}, \mathrm{H} 8\right)$.

${ }^{13}$ C NMR spectrum: 161.5 (C4a); 155.1 (C5a); 147.1 (C3); 143.6 (C1); 129.3 (C7); 124.5 (C8); 122.1 (C9); 119.7 (C9b); 118.8 (C9b); 112.0 (C6); 107.6 (C4).

IR spectrum: 1589; 1561;1457m;1428;1331m;1294m;1261m;1233m;1186; $1152 \mathrm{~m} ; 1016 \mathrm{w} ; 995 \mathrm{w} ; 947 ; 842 \mathrm{~m} ; 829 ; 755 ; 614 \mathrm{~m} ; 520 \mathrm{w} ; 434 \mathrm{w}$.

\section{5 [1]Benzofuro[3,2-c]pyridine (V)}

Zinc powder $(12.07 \mathrm{~g} ; 185 \mathrm{mmol})$, was added to $I V(9.0 \mathrm{~g} ; 44 \mathrm{mmol})$ and acetic acid $\left(15 \mathrm{~cm}^{3}\right)$ and mixture was refluxed for $8 \mathrm{~h}$, then filtered and the solvent was distilled off under reduced pressure. The residue was alkalized with diluted sodium hydroxide solution and extracted with chloroform. The solution was dried with sodium sulfate and the solvent was evaporated. Yield: $4.65 \mathrm{~g}(62.5 \%)$, m.p. $74-76{ }^{\circ} \mathrm{C}$ (hexane) white crystals.

For $\mathrm{C}_{11} \mathrm{H}_{7} \mathrm{NO}\left(M_{r}=169.18\right) w_{\mathrm{i}}$ (calc.): $78.09 \% \mathrm{C}, 4.17 \% \mathrm{H}, 8.28 \% \mathrm{~N}$; $w_{\mathrm{i}}$ (found): $78.12 \% \mathrm{C}, 4.01 \% \mathrm{H}, 8.16 \% \mathrm{~N}$.

${ }^{1} \mathrm{H}$ NMR spectrum: $9.42\left(\mathrm{~d},{ }^{5} J(1,4)=0.88 \mathrm{~Hz}, \mathrm{H} 1\right) ; 8.65\left(\mathrm{~d},{ }^{3} J(3,4)=5.8 \mathrm{~Hz}, \mathrm{H} 3\right)$; $8.25\left(\mathrm{dd},{ }^{3} J(9,8)=7.6 \mathrm{~Hz},{ }^{4} J(9,7)=1.2 \mathrm{~Hz}, \mathrm{H} 9\right) ; 7.785\left(\mathrm{dd},{ }^{3} J(6,7)=8.2 \mathrm{~Hz},{ }^{5} J(6,9)\right.$ $=0.88 \mathrm{~Hz}, \mathrm{H} 6) ; 7.78\left(\mathrm{dd},{ }^{3} J(4,3)=5.8 \mathrm{~Hz},{ }^{5} \mathrm{~J}(4,1)=0.88 \mathrm{~Hz}, \mathrm{H} 4\right) ; 7.60\left(\mathrm{t},{ }^{3} J(7,6)=\right.$ $\left.8.2 \mathrm{~Hz},{ }^{4} \mathrm{~J}(7,9)=1.2 \mathrm{~Hz}, \mathrm{H} 7\right) ; 7.49\left(\mathrm{t},{ }^{3} \mathrm{~J}(8,7)=7.6 \mathrm{~Hz},{ }^{4} \mathrm{~J}(8,6)=0.88 \mathrm{~Hz}, \mathrm{H} 8\right)$.

${ }^{13} \mathrm{C}$ NMR spectrum: 160.4 (C4a); 155.7 (C5a); $147.6(\mathrm{C} 3) ; 143.9$ (C1); 128.6 (C7); 124.1 (C8); 121.5 (C9); 121.1 (C9b); 120.7 (C9a); 111.9 (C6); 107.5 (C4).

IR spectrum: $3063-3037 ; 1590 ; 1577 ; 1481 \mathrm{~m} ; 1463 ; 1446 ; 1331 \mathrm{~m} ; 1293 \mathrm{~m} ; 1266 \mathrm{~m}$; 1243w; 1207; 1186; 1162; 1106m; 1009m; 995m; 862; 838; 823; 776; 751; 734; 595.

\section{6 [1]Benzofuro[3,2-c]pyridin-2-oxide (VI)}

A mixture of $V(1.42 \mathrm{~g} ; 8.4 \mathrm{mmol})$ and 3-chloroperoxybenzoic acid (70\%, $3.69 \mathrm{~g}$; $14.6 \mathrm{mmol})$ in dichloromethane $\left(50 \mathrm{~cm}^{3}\right)$ was stirred at room temperature for $4 \mathrm{~h}$. The 
reaction mixture was filtered and extracted with sodium carbonate $(10 \%)$, and water. The organic layer was dried over sodium sulfate, filtered and solvent was evaporated and residue was crystallized. Yield: 1.01 g, 65\%., m.p. 205-206 ${ }^{\circ} \mathrm{C}$ (toluene).

For $\mathrm{C}_{11} \mathrm{H}_{7} \mathrm{NO}_{2}\left(M_{r}=185.18\right) w_{\mathrm{i}}$ (calc.): $71.35 \% \mathrm{C}, 3.81 \% \mathrm{H}, 7.56 \% \mathrm{~N}$; $w_{\mathrm{i}}$ (found): $71.42 \% \mathrm{C}, 3.79 \% \mathrm{H}, 7.38 \% \mathrm{~N}$.

${ }^{13} \mathrm{C}$ NMR spectrum: $9.21\left(\mathrm{~d},{ }^{4} J(1,3)=1.8 \mathrm{~Hz}, \mathrm{H} 1\right) ; 8.32\left(\mathrm{dd},{ }^{3} J(3,4)=7.1 \mathrm{~Hz},{ }^{4} J(3,1)=\right.$ $1.8 \mathrm{~Hz}, \mathrm{H} 3) ; 8.20\left(\mathrm{dd},{ }^{3} J(9,8)=7.6 \mathrm{~Hz},{ }^{4} J(9,7)=1.4 \mathrm{~Hz}, \mathrm{H} 9\right) ; 7.82\left(\mathrm{~d},{ }^{3} J(4,3)=7.1\right.$ $\mathrm{Hz}, \mathrm{H} 4) ; 7.76\left(\mathrm{dd},{ }^{3} J(6,7)=8.2 \mathrm{~Hz},{ }^{4} J(6,8)=1.7 \mathrm{~Hz}, \mathrm{H} 6\right) ; 7.64\left(\mathrm{t},{ }^{3} J(7,6)=8.2 \mathrm{~Hz}\right.$, $\left.{ }^{4} J(7,9)=1.4 \mathrm{~Hz}, \mathrm{H} 7\right) ; 7.48\left(\mathrm{t},{ }^{3} J(8,9)=7.6 \mathrm{~Hz},{ }^{4} J(8,6)=1.7 \mathrm{~Hz}, \mathrm{H} 8\right)$.

${ }^{13} \mathrm{C}$ NMR spectrum: 156.8 (C5a); 151.3 (C4a); 138.3 (C3); 132.6 (C1); 129.7 (C7); 124.2 (C6); 123.0 (C9b); 122.5 (C9); 120.2 (C9a); 112.2 (C8); 109.9 (C4).

IR spectrum: $1661 ; 1470 ; 1440 ; 1308 \mathrm{~m} ; 1288 ; 1205 ; 1158 ; 1098 ; 1010 \mathrm{~m} ; 929 \mathrm{~m} ; 828$; $769 ; 742 ; 596 ; 526 ; 425$.

\section{7 [1]Benzofuro[3,2-c]pyridine-1-carbonitrile (VII)}

To solution of potassium cyanide $(1.25 \mathrm{~g}, 19 \mathrm{mmol})$ in water $\left(2 \mathrm{~cm}^{3}\right)$ a solution of [1]benzofuro[3,2-c]pyridin-2-oxide $(V I)(3.42 \mathrm{~g}, 1.85 \mathrm{mmol})$ in dichloromethane (10 $\left.\mathrm{cm}^{3}\right)$ was added and then dropwise a solution of benzoyl chloride $\left(0.3 \mathrm{~cm}^{3}, 2.15\right.$ $\mathrm{mmol})$ in dichloromethane $\left(10 \mathrm{~cm}^{3}\right)$. After vigorous stirring at room temperature for 2 days, the organic layer of the reaction mixture was separated and aqueous layer was extracted with chloroform. After drying over magnesium sulfate, the combined organic layers were evaporated and residue was purified by column chromatography on silica gel (hexane-ethyl acetate $3: 1$ ). The obtained VII was crystallized. Yield: 3.05g, (85\%), m.p. 135-137 ${ }^{\circ} \mathrm{C}$ (hexane) 139-141 ${ }^{\circ} \mathrm{C}$ (BENCKOVÁ and KRUTOŠÍKOVÁ, 1999).

For $\mathrm{C}_{12} \mathrm{H}_{6} \mathrm{~N}_{2} \mathrm{O}\left(M_{r}=194.19\right) w_{\mathrm{i}}$ (calc.): $74.22 \% \mathrm{C}, 3.11 \% \mathrm{H}, 14.43 \% \mathrm{~N} ; w_{\mathrm{i}}$ (found): $74.32 \% \mathrm{C}, 3.18 \% \mathrm{H}, 14.36 \% \mathrm{~N}$.

${ }^{13} \mathrm{C}$ NMR spectrum: $8.81\left(\mathrm{~d},{ }^{3} J(3,4)=5.57 \mathrm{~Hz}, \mathrm{H} 3\right) ; 8.21\left(\mathrm{~d},{ }^{3} J(9,8)=7.63 \mathrm{~Hz}, \mathrm{H} 9\right)$; $8.14\left(\mathrm{~d},{ }^{3} J(4,3)=5.57 \mathrm{~Hz}, \mathrm{H} 4\right) ; 7.89\left(\mathrm{~d},{ }^{3} \mathrm{~J}(6,7)=8.5 \mathrm{~Hz}, \mathrm{H} 6\right) ; 7.76\left(\mathrm{t},{ }^{3} \mathrm{~J}(7,6)=7.92\right.$ $\left.\mathrm{Hz},{ }^{3} J(7,8)=7.63 \mathrm{~Hz}, \mathrm{H} 7\right) ; 7.62\left(\left(\mathrm{t},{ }^{3} J(8,7)=7.63 \mathrm{~Hz},{ }^{3} J(8,9)=7.63 \mathrm{~Hz}, \mathrm{H} 8\right)\right.$.

${ }^{13} \mathrm{C}$ NMR spectrum: 160.4 (C4a); 155.8 (C5a); 148.8 (C3); 130.9 (C7); 125.0 (C8); 124.9 (C9b); 124.2 (C1); 121.4 (C9); 118.4 (C9a); 116.2 (CN); 112.6 (C6); 111.4 (C4).

IR spectrum: 3093; 2227m;1627w; 1591; 1567; 1483w; 1458; 1422; 1336; 1295w; $1263 ; 1231 ; 1182 ; 1109 ; 1072 \mathrm{w} ; 1015 \mathrm{~m} ; 1005 ; 944 \mathrm{w} ; 841 ; 806 ; 784 \mathrm{w} ; 748 ; 641 \mathrm{w}$; $568 \mathrm{w} ; 543 \mathrm{~m}$.

\subsection{Tetra- $\mu$-acetato-bis[([1]benzofuro[3,2-c]pyridine)copper(II)] (VIII)}

To a $\mathrm{Cu}\left(\mathrm{CH}_{3} \mathrm{CO}_{2}\right)_{2} . \mathrm{H}_{2} \mathrm{O}(1.5 \mathrm{mmol})$ in ethanol $\left(5 \mathrm{~cm}^{3}\right)$ was added the solution of the title compound $(3.2 \mathrm{mmol})$ in ethanol $\left(2 \mathrm{~cm}^{3}\right)$. Small blue-green crystals were 
collected after $2 \mathrm{~d}$. These were filtered off, washed with ethanol and crystallized from tetrahydrofuran.

For $\mathrm{C}_{30} \mathrm{H}_{26} \mathrm{~N}_{2} \mathrm{Cu}_{2} \mathrm{O}_{10}\left(M_{r}=701.63\right) w_{\mathrm{i}}$ (calc.): $51.35 \% \mathrm{C}, 3.73 \% \mathrm{H}, 3.99 \% \mathrm{~N}, 18.11 \%$ $\mathrm{Cu} ; w_{\mathrm{i}}$ (found): $51.43 \% \mathrm{C}, 3.65 \% \mathrm{H}, 3.99 \% \mathrm{~N}, 18.15 \% \mathrm{Cu}$.

IR spectrum: 3852; 3820; 3674; 3628; 3441; 3085; 3053; 2569; 2527; 1633; 1605; $1592 ; 1485 ; 1466 ; 1448 ; 1333 ; 1292 ; 1251 ; 1212 ; 1177 ; 1154 ; 1146 ; 1041 ; 1024 ; 878$; $838 ; 821 ; 774 ; 758 ; 596 ; 574 \mathrm{w} ; 562 \mathrm{w} ; 523 \mathrm{w}$.

\subsection{Bis([1]benzofuro[3,2-c]pyridine- $\kappa N)$ dichloridocobalt(II) (IX)}

To a $\mathrm{CoCl}_{2} \cdot 6 \mathrm{H}_{2} \mathrm{O}(1 \mathrm{mmol})$ solution in ethanol $\left(4 \mathrm{~cm}^{3}\right)$ was added the solution of $V$ ( $3 \mathrm{mmol}$ ) in ethanol $\left(4 \mathrm{~cm}^{3}\right)$ at room temperature. Small blue crystals were formed after $3 \mathrm{~d}$, which were filtered off, washed with ethanol and crystallized from tetrahydrofuran.

For $\mathrm{C}_{22} \mathrm{H}_{14} \mathrm{Cl}_{2} \mathrm{CoN}_{2} \mathrm{O}_{2}\left(M_{r}=468.21\right) w_{\mathrm{i}}$ (calc.): $56.43 \% \mathrm{C}, 3.01 \% \mathrm{H}, 5.98 \% \mathrm{~N}, 12.58$ $\mathrm{Co}$; $w_{\mathrm{i}}$ (found): $56.67 \% \mathrm{C}, 2.96 \% \mathrm{H}, 5.96 \% \mathrm{~N}, 11.96 \mathrm{Co}$.

IR spectrum: $1626 ; 1596 ; 1484 \mathrm{~m} ; 1484 ; 1467 ; 1450 ; 1433 ; 1210 \mathrm{~m} ; 1187 ; 1165$; $1018 \mathrm{w} ; 872 ; 840 \mathrm{~m} ; 777 ; 757 \mathrm{~m} ; 681 \mathrm{~m} ; 629 ; 598 \mathrm{w} ; 567 \mathrm{w} ; 523 \mathrm{w}$.

\section{Results and discussion}

(E)-3-(1-Benzofuran-2-yl)propenoic acid (I) was prepared from 1-benzofuran-2carbaldehyde under the Doebner's conditions. The obtained acid $I$ was converted to the corresponding azide II, which was cyclized by heating in diphenyl ether to [1]benzofuro[3,2-c]pyridin-1(2H)-one (III). This compound was aromatized with phosphorus oxychloride to chloroderivative $I V$ which was reduced with zinc and acetic acid to the title compound $V$. [1]Benzofuro[3,2-c]pyridin-2-oxide $(V I)$ was synthesized by reaction of $V$ with 3-chloroperoxybenzoic acid in dichloromethane. Treatment $V I$ with benzoyl chloride and potassium cyanide (Reissert-Henze reaction) was shown to produce the corresponding [1]benzofuro[3,2-c]pyridine-1-carbonitrile (VII). This transformation was easily verified by means of infrared spectra, loss of $N$-oxide band at $1205 \mathrm{~cm}^{-1}$ in the spectra of VII and appearance of cyano group absorption at 2227 $\mathrm{cm}^{-1}$. Disappearance of the most downfield signal in the ${ }^{1} \mathrm{H}$ NMR spectrum (at 9.21 ppm) of the starting compound VI also supported the structure of the compound VII. For the assignment of carbon signals in the synthesized compounds 2D-spectra,gCOSY, gHSQCAD and gHMBCAD were used.

\section{Conclusions}

The title compound [1]benzofuro[3,2-c]pyridine (V) was prepared five step synthesis and was used for preparation of complex compounds. The structures of VIII, $I X$ were proved by X-ray analysis (Figures 1,2 ). The structural data are presented in (VRABEL, 2007). 


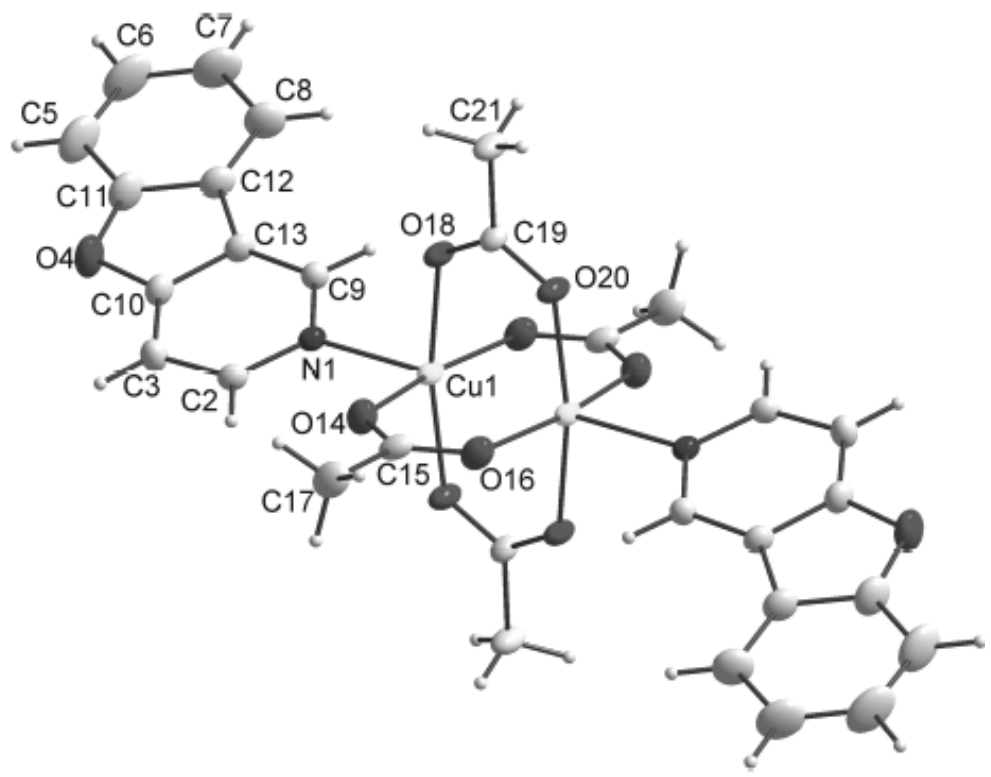

Fig. 1. The molecular structure of the complex compound VIII (VRABEL et al. 2007).

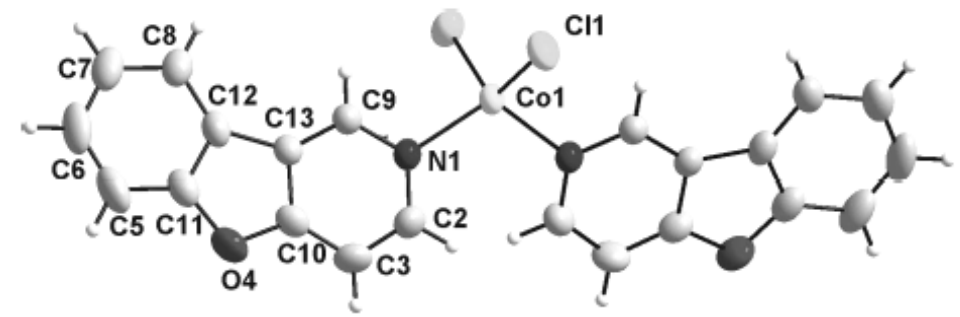

Fig. 2. The molecular structure of the complex compound IX (VRABEL et al. 2007).

Acknowledgements: This work was supported by the Grant Agency of the Ministry of Education of the Slovak Republic VEGA (Grant No. 1/3584/06). NMR experimental part of this work was facilitated by support of Slovak National Research and Development Program No. 2003SP200280203.

\section{References}

BARAN, P., BOČA, M., BOČA, R., KRUTOŠÍKOVÁ, A., MIKLOVIČ, J., PELIKÁN, J., TITIŠ, J.: Structural Characterization, Spectral and Magnetic Properties of Isothiocyanate Nickel(II) Complexes with Furopyridine Derivatives. Polyhedron, 24, 2005, 1510-1516. 
BENCKOVÁ, M., KRUTOŠÍKOVÁ, A.: Synthesis of pyrrolo[2',3':4,5]furo[3,2c]pyridines. Monatsh. Chem., 126, 1995, 753-758.

BENCKOVÁ, M., KRUTOŠÍKOVÁ, A.: 5-Aminofuro[3,2-c]pyridinium Tosylates and Substituted Furo[3,2-c]pyridine- $N$-Oxides: Synthesis and Reactions. Collect. Czech. Chem. Commun., 64, 1999, 539-547.

BOBOŠÍK, V., KRUTOŠÍKOVÁ, A., JORDIS, U.: Synthesis and Reactions of 2,3Dimethyfuro[3,2-c]pyridines. Monatsh. Chem., 126, 1995, 747-752.

KRUTOŠÍKOVÁ, A., DANDÁROVÁ, M., CHYLOVÁ, J., VÉGH, D.: Condensed O$N$-Heterocycles by the Transformation of Azidoacrylates. Monatsh. Chem., 123, 1992, 807-815.

KRUTOŠÍKOVÁ, A., DANDÁROVÁ, M., ALFÖLDI, J.: Substituted Vinyl Azides in the Synthesis of Condensed Nitrogen Heterocycles. Chem. Papers, 48, 1994, 268273.

KRUTOŠÍKOVÁ, A., SLEZIAK, R: Synthesis of 2-Arylfuro[3,2-c]pyridines and their Derivatives. Collect. Czech. Chem. Commun., 61, 1996, 1627-1636.

KRUTOŠÍKOVÁ, A., MITASOVÁ, B., JÓNA, E., BOBOŠÍKOVÁ, M.: Synthesis, Thermal and Spectral Properties of $\mathrm{Cu}(\mathrm{II})$ and $\mathrm{Ni}(\mathrm{II})$ Complexes with Furopyridines or Quinoline. Chem. Papers, 55, 2001, 290-293.

MIKLOVIČ, J., KRUTOŠÍKOVÁ, A., BARAN, P.: Two Furopyridine Complexes of Nickel(II) Isothiocyanate. Acta Cryst., C60, 2004, m227-m230.

MOJUMDAR, S.C., MIKLOVIČ, J., KRUTOŠíKOVÁ, A., VALIGURA, D., STEWART, J. M.: Furopyridines and Furopyridine-(Ni(II) Complexes: Synthesis, Thermal and Spectral Characterization. J. Therm. Anal. Cal., 81, 2005, 211-215.

SHERMAN, A.R.: Bicyclic 5-6 Systems: Two Heteroatoms 1:1. In: C.A. RAMSDEN (Ed.) Comprehensive Heterocyclic Chemistry II. Pergamon Press, Oxford, 1996, 167-227.

VRÁBEL, V., ŠVORC, L., JURISTOVÁ, N., MIKLOVIČ, J., KOŽÍǏEK, J.: Tetra- $\mu$ acetato-bis[(benzofuro[3,2-c]pyridine]copper(II). Acta Cryst., E63, 2007, m2112$\mathrm{m} 2113$.

VRÁBEL, V., ŠVORC, L., JURISTOVÁ, N., MIKLOVIČ, J., KOŽÍŠEK, J.: Bis(1benzofuro[3,2-c]pyridine- $\kappa N$ )dichloridocobalt(II). Acta Cryst., E63, 2007, m24272428 . 